\title{
Prevalencia de hipertensión arterial en población mayor de 65 años ingresada en un Servicio de Medicina Interna
}

\author{
S. CINZA SANJURJO, A. CABARCOS ORTIZ DE BARRÓN ${ }^{1}$ E. NIETO POL, \\ J. A. TORRE CARBALLADA ${ }^{1}$ \\ Servicio de Atención Primaria Concepción Arenal. ' Servicio de Medicina Interna. \\ Hospital Clínico Universitario. Santiago de Compostela. A Coruña
}

INCIDENCE OF HYPERTENSION IN ELDERLY PATIENTS INTAKED

ON INTERNAL MEDICINE DEPARTMENT

\section{RESUMEN}

Objetivos: Conocer la prevalencia de hipertensión arterial y de hipertensión sistólica aislada en los pacientes mayores de 65 años ingresados en un Servicio de Medicina Interna.

Material y métodos: Estudio descriptivo trasversal, en el que se incluyeron los pacientes mayores de 65 años que ingresaron durante el año 2002 en el Servicio de Medicina Interna del Hospital Clínico Universitario de Santiago de Compostela. Las variables recogidas fueron: sexo, edad, tiempo de ingreso, factores de riesgo cardiovascular, cifras de presión arterial (PA), cifras de glucemia, cifras de colesterolemia, patología cardiovascular coexistente, exitus intrahospitalario y tratamientos al alta. Se emplearon índices estadísticos descriptivos de variables cualitativas y cuantitativas, Chi-cuadrado, Mann-Withney-Wilcoxon y Regresión logística.

Resultados: Se obtuvo una población de 770 pacientes. La prevalencia de hipertensión arterial (HTA) fue de 37,0\% y la de hipertensión sistólica aislada (HSA) de 25,9\%. La prevalencia de HSA es mayor en los pacientes con mal control de PA (67,5 vs. 8,9\%), p <0,01. El 78\% de los pacientes hipertensos presentaron asociado al menos un factor de riesgo cardiovascular y el 60,9\% presentaron asociada alguna patología cardiovascular. Se encontró en los pacientes hipertensos una mayor prevalencia de diabetes mellitus (66,7 vs. 58,8\%), hipercolesterolemia (15,8 frente $5,5 \%)$ y de cardiopatía isquémica $(21,7 \%$ frente $13,0 \%)$ que en los que tenían una HSA. Se administró tratamiento farmacológico en el 49,8\% de los HTA y en el $61,8 \%$ de los HSA. No hubo diferencias entre los dos grupos en cuanto al uso de fármacos antihipertensivos, salvo en el grupo de diuréticos de asa $(41,2$ frente a $30,2 \%)$ e IECA (17,6 frente a 9,5\%), que se emplearon más en los HSA.

Conclusiones: Las prevalencias de HTA y de HSA son bajas para el grupo de población estudiado. La prevalencia de cardiopatía isquémica es superior en pacientes con HTA que HSA, y es la patología cardiovascular asociada a mayor mortalidad en hipertensos. El tratamiento farmacológico empleado en la HSA y en los pacientes con otras patologías concomitantes no se adecúan en general a las recomendaciones de los actuales consensos.

PALABRAS CLAVE: Hipertensión arterial. Hipertensión sistólica aislada. Prevalencia. Mayores 65 años. Medicina Interna.

\section{ABSTRACT}

Objetives: To know the arterial hypertension prevalence and isolated systolic hypertension in elderly patients intaked on internal medicine department.

Material and methods: A cross-sectional study of elderly hypertensive patients intaked between 1 to January 2002 to 31 December 2002 in a Internal Medicine Service of the Hospital Clinico Universitario de Santiago de Compostela. The recorded variables were: sex, age, number of days in hospital, hospital exitus, cardiovascular risk factors, number of blood pressure, glucose, cholesterolemia, cardiovascular disease and treatments. It was used descriptive statistic rates of quantitative and qualitative variables, Mann-Withney-Wilcoxon and logistic regresion.

Results: The study included 770 patients. The arterial hypertension (HTA) prevalence was 37\% and the isolated systolic hypertension (ISH) prevalence were $25.9 \%$. The ISH prevalence was greater in the patients with bad control of blood pressures (67.5 vs. 8.9\%), $p<0.01$. The $78 \%$ of patients with hypertension showed one or more risk cardiovascular factor and the $60.9 \%$ of these patients showed one or more cardiovascular disease. We found in patients with hypertension bigger prevalence of mellitus diabetes (66.7 vs. 58.8\%), hypercholesterolemia (15.8 vs. 5.5\%) and cardiac disease (21.7 vs. $13.0 \%)$ than in patients with HAS. It was ran farmacologic treatment in the $49.8 \%$ of the patients with high blood pressure and in the $61.8 \%$ of the patients with isolated systolic hypertension. The loop diuretics (41.2 vs. 30.2\%) and ECAI (17.6 vs. 9.5\%) were more used in isolated systolic hypertension.

Conclusions: The hypertension and isolated systolic hypertension prevalences are small in the people studied. The prevalence of heart disease is bigger in patients with high blood pressure, and also it is the disease that caused more deaths in hypertensive patients. The pharmacological treatment used in hypertensive patients is not the most guarantied by the actually evidence.

KEY WORDS: Hypertension. Internal Medicine. Aged. Prevalence.

Cinza Sanjurjo S, Cabarcos Ortiz de Barrón A, Nieto Pol E, Torre Carballada JA. Prevalencia de hipertensión arterial en población mayor de 65 años ingresada en un Servicio de Medicina Interna. An Med Interna (Madrid) 2006; 23: 577-581.

Trabajo aceptado: 26 de julio de 2006

Correspondencia: Sergio Cinza Sanjurjo. C/ Ramón Cabanillas, 10, $7^{\circ}$ C, 15701 Santiago de Compostela (A Coruña). Fax 981536594. e-mail: sergiocinza@mundo-r.com 


\section{INTRODUCCIÓN}

El aumento de la esperanza de vida de la población y el incremento de la presión arterial (PA) asociado a la edad está originando que la hipertensión arterial (HTA) sea una de los factores de riesgo cardiovascular más prevalentes en nuestro entorno, superando el $65 \%$ en mayores de 60 años (1), porcentaje que previsiblemente seguirá aumentando en los próximos años (2). Es conocido que la presión arterial sistólica (PAS) aumenta progresivamente con la edad, mientras que la presión arterial diastólica (PAD) a partir de los 50 a 60 años tiende a disminuir (3), lo que origina una mayor prevalencia de HSA en la población anciana. Como resultado del estudio Framingham (4), se conoce que la PAS es un marcador de mortalidad cardiovascular y de mortalidad global más importante que la PAD $(5,6)$; a pesar de ello, la HSA sigue sin recibir el reconocimiento que se merece por parte de los clínicos como factor de riesgo cardiovascular, ya que en muchas ocasiones no se administra el tratamiento adecuado (7).

A una mayor prevalencia de HSA asociada a la edad, también concurren una mayor incidencia de factores de riesgo cardiovascular y una elevada morbi-mortalidad cardiovascu$\operatorname{lar}(8,9)$.

Actualmente, sólo un tercio de los hipertensos que reciben tratamiento farmacológico cumplen los criterios de control de PA $(1,10)$. Existen múltiples factores que influyen en este mal control $(1,11)$ : medición de la PA, variabilidad de las cifras de PA a lo largo del día, toma de medicación por parte del paciente, tratamiento farmacológico habitual en forma de monoterapia, elevado índice de masa corporal (IMC) y no haber realizado visitas a su médico de cabecera en el último año.

Por todo lo expuesto anteriormente, se planteó el presente estudio con el objetivo principal de conocer la prevalencia de hipertensión arterial y de hipertensión sistólica aislada en los pacientes mayores de 65 años ingresados en un Servicio de Medicina Interna y el tratamiento administrado.

\section{MATERIAL Y MÉTODOS}

\section{DISEÑO DEL ESTUDIO}

Se trata de un estudio descriptivo trasversal, en el que se incluyeron los pacientes mayores de 65 años ingresados durante el año 2002 en el Servicio de Medicina Interna del Hospital Clínico Universitario de Santiago de Compostela. Se incluyeron un total de 770 pacientes, de los que se revisaron los informes de alta y posteriormente la historia clínica.

Las variables recogidas para cada paciente fueron: sexo, edad, tiempo de ingreso, factores de riesgo cardiovascular, valores de PA, niveles de glucemia, niveles de colesterolemia, patología cardiovascular, exitus intrahospitalario y tratamiento en el momento del alta.

\section{DEFINICIÓN DE CRITERIOS}

Tabaquismo: se consideraba fumador a aquella persona que en el último mes previo al ingreso consumía tabaco (cigarros, puros y pipa), al menos una unidad a lo largo del mes. Se consideraba exfumador al paciente que no había fumado en el último año (12).
Consumo de alcohol: Se consideraba consumidor de alcohol al paciente que superaba la cantidad de una copa de vino diaria (13).

Hipertensión arterial: diagnóstico previo. Confirmada o diagnosticada al ingreso, según criterios del Joint National Comittee VII (JNC-VII) (14), registrada en condiciones estandarizadas, adaptando las cifras aconsejadas de PA en grupos especiales de riesgo (Diabetes Mellitus, Insuficiencia Renal, etc.) (15).

Hipertensión sistólica aislada: Según criterios del JNCVII (14), cuando presentaba cifras de PAS iguales o superiores a $140 \mathrm{mmHg}$ y PAD inferior a $90 \mathrm{mmHg}$ en el momento del diagnóstico de HTA.

Presión de pulso: la diferencia entre la PAS y la PAD expresada en $\mathrm{mmHg}(16)$.

Hipercolesterolemia: diagnóstico previo. Confirmada o diagnosticada al ingreso, según criterios del Third Report of the National Cholesterol Education Program (NCEP) - Adult Treatment Panel III (ATP-III) (17), cuando la cifra media de colesterol total en dos determinaciones era superior a 250 $\mathrm{mg} / \mathrm{dl}$, en prevención primaria, y superior a $200 \mathrm{mg} / \mathrm{dl}$, en prevención secundaria.

Diabetes Mellitus: diagnóstico previo. Confirmada o diagnosticada al ingreso, según criterios de la Asociación Americana de Diabetes (18).

Factor de riesgo cardiovascular: si entre los antecedentes personales o entre los diagnósticos al alta presentaba HTA, diabetes mellitus, hipercolesterolemia, consumo de tabaco y/o consumo de alcohol (14).

Patología cardiovascular: si entre los antecedentes personales o en los diagnósticos al alta presentaba cardiopatía isquémica, insuficiencia cardíaca, accidente cerebrovascular o arteriopatía periférica (14).

\section{ANÁLISIS ESTADÍSTICO}

Los datos recogidos se incluyeron en una base de datos diseñada para tal efecto en Excel 2003, y fueron analizados posteriormente empleando el paquete estadístico G-STAT versión 2.0.

Para el análisis univariante se emplearon índices estadísticos descriptivos de variables cualitativas y cuantitativas. Para el análisis bivariante de variables cualitativas se empleó Chicuadrado; en caso de variables cuantitativas se empleó MannWhitney-Wilcoxon.

\section{RESULTADOS}

Se registraron un total de 770 pacientes que cumplían los criterios de inclusión para el estudio. Las características demográficas de la población se presentan en la Tabla I. Según los criterios planteados $(14,15)$, se diagnosticaron de hipertensos un total de 484 pacientes (62,9\%, IC 95\%: 59,4\% - 66,3\%) de los cuales 285 pacientes presentaban HTA (37,0\%, IC 95\%: 33,5\% - 40,5\%) y 199 presentaban HSA $(25,9 \%$, IC 95\%: $22,7 \%-29,0 \%)$. Se observó un predominio de sexo femenino en el grupo de pacientes hipertensos $(53,9 \%$, IC 95\%: 50,3\% - 57,5\%), p = 0,04. No se observaron diferencias por sexo ni por edad entre los grupos con HTA y con HSA. 


\begin{tabular}{|c|c|c|}
\hline \multicolumn{3}{|c|}{ TABLA I } \\
\hline \multicolumn{3}{|c|}{$\begin{array}{c}\text { CARACTERÍSTICAS SOCIODEMOGRÁFICAS DE LA } \\
\text { POBLACIÓN DE ESTUDIO }\end{array}$} \\
\hline$n$ & $\%$ & \\
\hline \multicolumn{3}{|l|}{ Sexo } \\
\hline Varones & 393 & $48,89 \%$ \\
\hline Mujeres & 376 & $51,11 \%$ \\
\hline \multicolumn{3}{|l|}{ Edad } \\
\hline 65 - 69 años & 97 & $12,63 \%$ \\
\hline $70-74$ años & 125 & $16,28 \%$ \\
\hline 75 - 79 años & 177 & $23,05 \%$ \\
\hline $80-84$ años & 194 & $25,26 \%$ \\
\hline $85-89$ años & 120 & $15,63 \%$ \\
\hline $90-94$ años & 45 & $5,86 \%$ \\
\hline 95 - 99 años & 8 & $1,04 \%$ \\
\hline 100 - 104 años & 1 & $0,13 \%$ \\
\hline 105 - 109 años & 1 & $0,13 \%$ \\
\hline
\end{tabular}

En el grupo de pacientes hipertensos, el $15,3 \%$ presentaban cifras elevadas de PAD y el 44,4\% no cumplían los objetivos para la PAS, circunstancia que explica la mayor prevalencia de HSA entre los pacientes con mal control de PA $(67,5 \%$ vs $8,9 \%$; $\mathrm{p}<0,01)$.

El porcentaje de pacientes con diagnóstico previo de hipertensión arterial fue superior en el grupo de HTA $(78,6 \%)$ que en el grupo de HSA (44,2\%), p < 0,01.

$\mathrm{El} 65 \%$ de los pacientes presentaban al menos un factor de riesgo cardiovascular (FRCV), la frecuencia de cada uno de ellos en la población se presenta en la Tabla II. Paralelamente, se analizó la existencia de múltiples factores de riesgo, ver Fig.1.

La asociación de algún FRCV en hipertensos era superior que en los sujetos normotensos (78\% vs 43\%; $\mathrm{p}<0,01)$. La diabetes mellitus y la hipercolesterolemia fueron los FRCV más prevalentes entre los hipertensos, 63,4\% y $11,6 \%$ respectivamente ( $\mathrm{p}<0,01$ y $\mathrm{p}=0,04$ respectivamente). En cambio no se encontraron diferencias entre ambos grupos en cuanto al consumo de tabaco ni alcohol; $\mathrm{p}=0,42$ y $\mathrm{p}=0,51$ respectivamente.

Si se analizan los dos grupos de hipertensos, se observa que la prevalencia de diabetes mellitus es similar en ambos $(66,7 \%$ en HTA y $58,8 \%$ en HSA, $p=0,07)$. En cambio, la hipercolesterolemia presenta mayor prevalencia en el grupo de HTA $(15,8 \%$ vs $5,5 \%$; p < 0,01). El consumo de alcohol y

\section{TABLA II}

\begin{tabular}{lcc}
\multicolumn{4}{c}{ TABLA II } \\
PREVALENCIA DE LOS FACTORES DE RIESGO \\
CARDIOVASCULAR EN LA POBLACIÓN DE ESTUDIO \\
\hline Factor de riesgo & $n$ & $\%$ \\
\hline HTA & 484 & $63 \%$ \\
Diabetes mellitus & 424 & $55 \%$ \\
Hipercolesterolemia & 76 & $10 \%$ \\
Fumadores activos & 32 & $4 \%$ \\
Ex-fumadores & 80 & $10 \%$ \\
Consumo alcohol & 50 & $6 \%$ \\
Ex-bebedores & 32 & $4 \%$ \\
\hline
\end{tabular}

tabaco también fue similar en ambos $(\mathrm{p}=0,09, \mathrm{p}=0,56$, respectivamente).

El 59\% de la población estudiada presentaba alguna patología cardiovascular asociada, sin que se observasen diferencias entre ambos grupos de hipertensos $(59,6 \%$ en HTA vs $62,8 \%$ en HSA; $p=0,07)$. La más prevalente fue la insuficiencia cardíaca (41,1\%, IC 95\%: 37,6\% - 44,7\%), seguido de los accidentes cerebro-vasculares (17,1\%; IC 95\%: 14,4\% $19,9 \%)$ y de la cardiopatía isquémica (16,0\%; IC 95\%: $13,5 \%$ - $18,8 \%$ ) y de la isquemia de extremidades inferiores $(3 \%$; IC 95\%: $2 \%-4,5 \%$ ).

La cardiopatía isquémica fue la patología cardiovascular más prevalente en el grupo HTA $(21,7 \%$ vs $13,0 \%$; p < 0,01). Además, fue la que se asoció con mayor mortalidad en pacientes hipertensos $(20,4 \%$ vs $11,1 \%, \mathrm{p}=0,02)$.

El $54,7 \%$ de los pacientes hipertensos precisaron tratamiento farmacológico para alcanzar un correcto control de PA, siendo superior la administración de fármacos en pacientes con HSA $(61,8 \%$ vs 49,8\%; p < 0,01). El análisis de los grupos terapéuticos empleados para el tratamiento de la hipertensión reveló que los diuréticos de asa y los inhibidores del enzima convertidor de angiotensina (IECA) se empleaban con más frecuencia en pacientes con HSA $(41,2 \%$ y $17,6 \%$ vs $30,1 \%$ y $9,5 \%$; $<<0,01$, en ambos casos).

El análisis que relacionó el empleo de grupos terapéuticos en función de la patología concomitante demostró que los grupos de IECA y ARA-II era similar en diabéticos que no diabéticos $(\mathrm{p}=0,34 \mathrm{y} \mathrm{p}=0,54$, respectivamente). Tampoco se observaron diferencias en el empleo de calcio-antagonistas en pacientes con HSA, $p=0,32$. Y, finalmente, no se observó una mayor prescripción de beta-bloqueantes en pacientes con insuficiencia cardíaca ni cardiopatía isquémica, $\mathrm{p}=0,70$ y $\mathrm{p}=0,63$ respectivamente.

\section{DISCUSIÓN}

En el presente estudio, la prevalencia de hipertensión arterial en mayores de 65 años alcanza el 62,9\% de la población

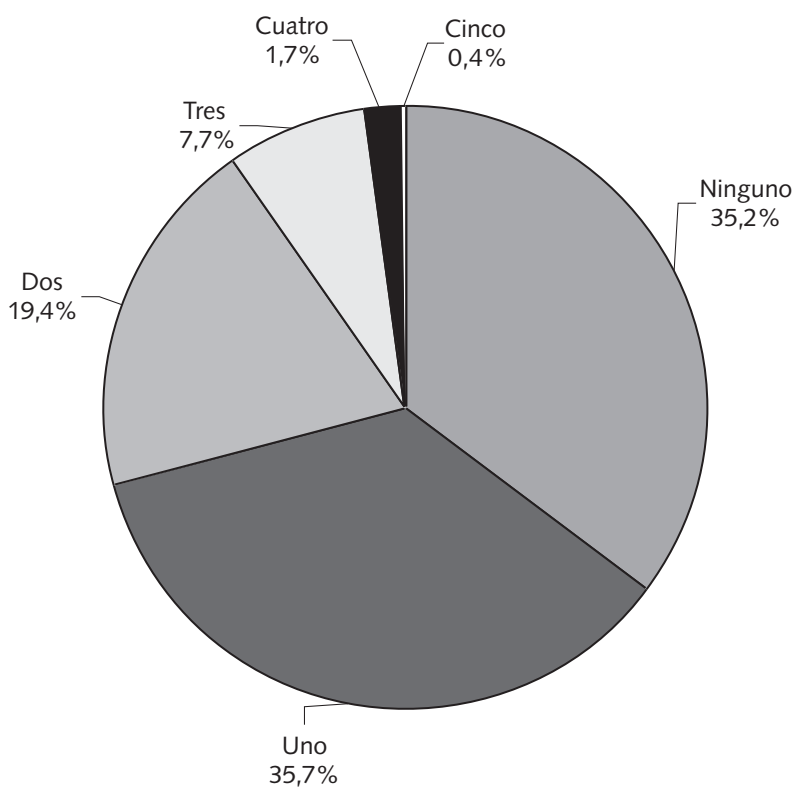

Fig. 1. Presencia de factores de riesgo cardiovascular asociados. 
de estudio y, particularmente, la HSA alcanza una prevalencia del 25,9\% (el 41,1\% de todos los hipertensos mayores de 65 años). Estas cifras de prevalencia son inferiores a las presentadas en estudios que emplean los mismos criterios diagnósticos (14), aunque sobre población no hospitalizada: el proyecto EPICARDIAN (19), en población mayor de 65 años, mostró una prevalencia de HTA de $67,7 \%$ y de HSA de $40,7 \%$; el estudio ECEHA (20), realizado en pacientes que acudían a centros de día y residencias de ancianos, mostró una prevalencia de HTA del 70,9\% y de HSA del 76,7\%.

Se ha observado una mayor prevalencia de HSA en pacientes con mal control de PA; además un porcentaje elevado de los pacientes con HSA no presentaban diagnóstico previo $(55,8 \%)$; ambas situaciones se relacionan probablemente con el hecho de que son pocos los facultativos que prestan atención al diagnóstico y tratamiento de la HSA (7). A pesar de ello, el control de PA era correcto en el 54,1\% de los pacientes tratados, cifra superior a la observada en la población hipertensa española de mayores de 65 años asistida en atención primaria (21).

La comorbilidad de DM e HTA obtenida en el presente trabajo (HTA e HSA: $66,7 \%$ y 58,8\% respectivamente) es superior a la registrada en el estudio Badalona (realizado sobre población general y centrado en esta asociación de factores de riesgo) (22), en el que se mostró una prevalencia próxima al $31 \%$ en ambos grupos.

El diagnóstico de hipercolesterolemia es muy inferior al de otros estudios (22-24), lo que muestra una escasa sensibilización en el Servicio de Medicina Interna de la importancia del mismo.

El estudio de la asociación de factores de riesgo demostró que el porcentaje de población susceptible de intervención es elevado, ya que el 64,9\% de los pacientes estudiados presentan al menos uno de los FRCV registrados en el estudio, a pesar de ello, esta cifra es inferior a la comunicada por otros autores (25).

La cardiopatía isquémica es la única patología cardiovascular que presenta diferencias entre ambos grupos de hipertensos, siendo mayor en los pacientes con HTA que con HSA, además se relaciona con una mayor mortalidad en el grupo de hipertensos.

Desde hace más de una década disponemos de evidencias sobre la necesidad de administrar tratamiento farmacológico en pacientes diagnosticados de HSA (26) para reducir la mortalidad cardiovascular $(22 \%)$, coronaria $(26 \%)$ y cerebro-vascular $(33 \%)$, siendo los calcio-antagonistas el grupo terapéutico más avalado (14). En el presente estudio, se observa mayor prescripción farmacológica en pacientes con HSA $(61,8 \%)$ que la notificada por otros autores (27) (56\%). También observamos que dicha prescripción es superior a la del resto de hipertensos $(61,8 \%$ vs $49,8 \%, \mathrm{p}<0,01)$, aunque no parece suficiente ya que no supera los dos tercios de los pacientes diagnosticados.

El análisis de prescripción de antihipertensivos según la comorbilidad asociada en los pacientes hipertensos ha demostrado que: 1) no existe mayor utilización de IECA (o ARA-II) o bien combinaciones de éstos con calcio-antagonistas en pacientes diabéticos a pesar de la demostrada nefroprotección que ofrecen (28), y que son recomendados en la última guía de la Asociación Americana de Diabetes (ADA) (29); 2) no existe mayor empleo de beta-bloqueantes en pacientes con insuficiencia cardíaca ni cardiopatía isquémica, a pesar de la evidencia existente de una importante reducción de la morbimortalidad (14) con su empleo.

Una limitación de nuestro estudio es la población seleccionada, por tratarse de población hospitalizada, lo que dificulta generalizar los resultados a la población general hipertensa de mayor edad. Sin embargo, ante los numerosos estudios similares realizados en población general, el principal objetivo era conocer la prevalencia de HTA y de HSA en la población ingresada en un servicio de medicina interna, lugar en el que en muchas ocasiones se realiza el diagnóstico y tratamiento inicial de la HTA y del resto de factores de riesgo cardiovascular, siendo la Atención Primaria la encargada del posterior seguimiento de estos pacientes.

En resumen, a modo de conclusión, se puede decir que las prevalencias encontradas de HTA $(62,9 \%)$ y de $\operatorname{HSA}(25,9 \%)$ son bajas teniendo en cuenta el grupo poblacional de que se trata y que el tratamiento farmacológico empleado en los pacientes hipertensos no es el más adecuado según la evidencia disponible.

Una interesante vía de investigación sería conocer cuál es la estrategia terapéutica con más posibilidades de efectividad y rentabilidad en Atención Primaria, para el control de los factores de riesgo cardiovascular tras el alta hospitalaria del paciente.

\section{Bibliografía}

1. Banegas JR, Rodríguez-Artalejo F, Ruilope LM, Graciano A, Luque M, De la Cruz-Troca JJ, et al. Hipertensión Magnitude and management in the elderly population of Spain. J Hypertens 2002; 20: 2157-64.

2. Chobanian AV, Bakris GL, Black HR, Cushman WC, Green LA, Izzo JL, et al. The Seventh Report of the Joint National Committee on Prevention, Detection, Evaluation and Treatment of High Blood Pressure: The JNC 7 Report. JAMA 2003; 289: 2560-72.

3. Burt VL, Cutler JA, Higgins M, Horan MJ, Labarthe D, Whelton P, et al. Trends in the prevalence, awareness, treatment, and control of hypertension in the adult US population: data from the health examination surveys, 1960 to 1991 . Hypertension 1995; 26: 60-9.

4. Kannel WB, Gordon T, Schwartz MJ. Systolic versus diastolic blood pressure and risk of coronary heart disease: Framingham Study. Am J Cardiol 1971; 27: 335-46.

5. National high blood pressure education program working group. Natio- nal high blood pressure education program working group report on hypertension in the elderly. Hypertension 1994; 23: 2175-85.

6. Sagie A, Larson MG, Levy D. The natural history of borderline isolated systolic hypertension. N Eng J Med 1993; 329: 1912-7.

7. Coppela WG, Whincup PH, Walker M, Ebrahim S. Identification and management of stroke risk in older people: a national survey of current practice in primary care. J Hum Hypertens 1997; 11: 185-91.

8. Lewington S, Clarke R, Qizilbash N, Peto R, Collins R. Prospective studies collaboration. Age-specific relevance of usual blood pressure to vascular mortality: a meta-analysis of individual data for one million adults in 61 prospective studies. Lancet 2002; 360: 1903-13.

9. Banegas JR, Rodríguez-Artejo F, De la Cruz JJ, De Andrés B, Rey J. Mortalidad relacionada con la hipertensión y la presión arterial en España. Med Clin (Barc) 1999; 112: 489-94.

10. Coca A. Evolución del control de la hipertensión arterial en España. 
Resultados del estudio Controlpres 2001. Hipertensión 2002; 19: 390-9.

11. O’Brien E, Asmar R, Beilin L, Imai Y, Mallion JM, Mancia G, et al, on behalf of the European Society of Hypertension Working Group on Blood Pressure Monitoring. European Society of Hypertension recommendations for conventional, ambulatory and home blood pressure measurement. J Hypertens 2003; 21: 821-48.

12. Doll R, Peto R, Wheatley K, Gray R, Sutherland I. Mortality in relation to smoking: 40 years' observational study on male British doctors. BMJ 1994; 309: 901-11.

13. Wannamethee SG, Shaper AG. Patterns of alcohol intake and risk of stroke in middle-aged British men. Stroke 1996; 27: 1033-9.

14. Joint National Committee on Prevention, Detection, Evaluation, and Treatment og High Blood Pressure. The Seventh report of the Joint National Committee on Prevention, Detection, Evaluation, and Treatment of High Blood Presure (JNC VII). JAMA 2003; 289: 2560-72.

15. 2003 European Society of Hypertension-European Society of Cardiology. Guidelines for the management of arterial hypertension. J Hypertens 2003; 21: 1011-53

16. Safar ME, Cloarec-Blauchard L, London GM. Arterial alterations in hypertension with a disproporcionate increase in systolic over diastolic blood pressure. J Hypertens 1996; 14 (Supl. 2): 103-9.

17. Executive Summary of the Third Report of the National Cholesterol Education Program (NCEP) Expert Panel on Detection, Evaluation and Treatment of High Blood Cholesterol in Adults (Adult Treatment Panel III). JAMA 2001; 281: 2486-97.

18. Expert Committee on the diagnosis and classification of Diabetes Mellitus. American Diabetes Association: clinical practice recommendations 2002. Diabetes Care 2002; 25 (Supl. 1): S1-147.

19. Gabriel Sánchez R, Alonso Arroyo M, Bermejo Pareja F, Muñiz García J, López Rodríguez I, Suárez Fernández C, et al. Proyecto EPICARDIAN: studio epidemiológico sobre enfermedades y factores de riesgo cardiovasculares en ancianos españoles. Diseño, método y resultados preliminares. Rev Esp Geriatr Gerontol 1996; 31: 327-34.

20. ECEHA. Estudio Cooperativo Español de Hipertensión Arterial en el anciano. Primera fase-prevalencia y características de la hipertensión arterial en el anciano en España. Sociedad Española de Geriatría y Gerontología. Liga Española para la lucha contra la HTA.
Sociedad Española de Cardiología. Sociedad Española de Medicina Familiar y Comunitaria. Madrid: Pharma Consult Services, SA. EDIPHARMA; 1996.

21. Rodríguez Roca GC, et al. Control de la hipertensión de los españoles $>65$ años asistidos en atención primaria. Rev Esp Cardiol 2005; 58: 359-66.

22. Albaladejo Blanco C, Martín García JA, López López I, Ródenas Aguilar JL, Ingla Mas J, López Vivo J. Grupos de riesgo cardiovascular y adecuación del tratamiento farmacológico en hipertensos de edad avanzada. Hipertensión 2001; 18: 305-13.

23. Jiménez C, Magaña JE, García Y, Díaz MA, Puig EM, Álvarez MC. ¿Estamos controlando el factor de riesgo "hipertensión arterial" en la población hipertensa de nuestro centro de salud? Hipertensión 1999; 16: 332-7.

24. González-Villalpando C, Stern MP, Haffner SM, González-Villalpando ME, Gaskill S, Rivera Martínez D. Prevalence of hypertension in a mexican population according to the Sixth report of the Joint Nacional Comittee on prevention, detection, evaluation and treatment of High Blood Pressure. J Cardiovasc Risk 1999; 6: 177-81.

25. Pineda Cuenca M, Custardoy Olavarrieta J, Ortín Arróniz JM, Cano Montoso JG, Andreu Ruiz MT, Grau C. Grado de conocimiento, tratamiento y control de la hipertensión arterial, hipercolesterolemia y diabetes mellitus en la población general adulta. Aten Primaria 2004; 33: 254-60.

26. SHEP Cooperative Research Group. Prevention of stroke by antihypertensive drug treatment in older persons with isolated systolic hypertension. JAMA 1991; 265: 3225-63.

27. López Fernández V, Peña Luengo LA, Iglesias Heredero A, Herrero Puente P, Vázquez Álvarez J, Suárez García S, et al. Prevalencia de hipertensión arterial sistólica aislada en una población mayor de 60 años. Hipertensión 2002; 19: 384-9.

28. Coca A, Ruilope LM. El bloqueo del sistema renina-angiotensina reduce la mortalidad y morbilidad cardiovascular atribuible a la hipertensión arterial en prevención primaria y secundaria. ¿El final del camino? Med Clin (Barc) 2000; 115; 78-180.

29. American Diabetes Association (ADA). Standard of medical Care for patients with Diabetes Mellitus. Diabetes Care 2002; 25 (Supl. 1): S33-49. 